Lysenko, O. \& Sneath, P. H. A. (1959). J. gen. Microbiol. 20, 284-290

\title{
The Use of Models in Bacterial Classification
}

\author{
BY O. LYSENKO \\ Laboratory of Insect Pathology, Czechoslovak Academy of Sciences, Institute \\ of Biology, Prague, Czechoslovakia \\ AND P. H. A. SNEATH
}

National Institute for Medical Research, Mill Hill, London, N.W. 7

SUMMARY: The making of models for taxonomic purposes and their use is described, and the fidelity with which they can represent the taxonomic relations between bacteria is discussed. These models are particularly useful for teaching purposes.

The use of equally weighted features in creating taxa allows the comparison of organisms by simple mathematical methods in which the similarity between two strains can be expressed as a percentage; the strains can then be grouped into taxa whose members show high similarity with each other. This method was described by Sneath $(1957 a, b)$, who used it for a study of the genus Chromobacterium. It also permits solid models to be made of taxonomic groups, in which the spatial arrangements of the component parts of a model is an expression of the relation of organisms or groups of organisms within the taxonomic group. The preparation of such models is the subject of the present paper.

\section{METHODS}

The meaning of the symbols used is given in Sneath (1957b). Two groups of bacteria were used for the models illustrated here. The first was the family Enterobacteriaceae as it is at present generally defined. The second was the group of chromobacteria studied by Sneath $(1957 b)$.

Enterobacteriaceae. In Table 1 are given the features currently used for the differentiation of a number of groups of the Enterobacteriaceae under names which are commonly given them. From Table 1 values of $S$ were calculated by the method of Sneath $(1957 b)$; these values are shown in Table 2. Since the data in Table 1 do not represent the features of single strains, a slightly different convention for obtaining $S$ was used;,$+(+)$ and $\times$ were counted as positive, and $d$ was counted as NC (no comparison). The data in Table 1 were taken mainly from the papers by Cowan (1956) and Kauffmann (1956), with supplementary information from Møller (1955) and Davis, Ewing \& Reavis (1957).

It should be noted that the tests used are selected, and therefore are biased; the use of these tests cannot show whether the groups are valid taxa, since this prejudges the question. They are used only as an illustration of the use of models, and the model is not a model of the ideal taxonomy of the family, but only of the current ideas about the taxonomy of the family. The model shown (Pl. 1, figs 1, 2) was made with distance $d$ proportional to $1-S$ (see below). 


\section{Table 2. $S$ values of Enterobacteriaceae}

Table 2 was derived from Table 1 as follows: for a particular comparison between two bacteria, $S=n_{s} /\left(n_{s}+n_{d}\right)$, where $n_{s}$ is the number of features for which they are both positive, and $n_{d}$ is the number of features for which one is positive and the other negative (and vice versa). The entries,$+(+)$ and $x$ in Table 1 were counted as positive, - was negative and d was counted as NC (no comparison made).

Group no.

$\begin{array}{llllllllllllll}1 & 2 & 3 & 4 & 5 & 6 & 7 & 8 & 9 & 10 & 11 & 12 & 13 & 14\end{array}$

Group name

Escherichia

Salmonella

Arizona

Citrobacter

Cloaca

Klebsiella

Hafnia

Serratia

Proteus vulgaris

P. mirabilis

Morganella

Providencia

Rettgerella

Shigella type A1

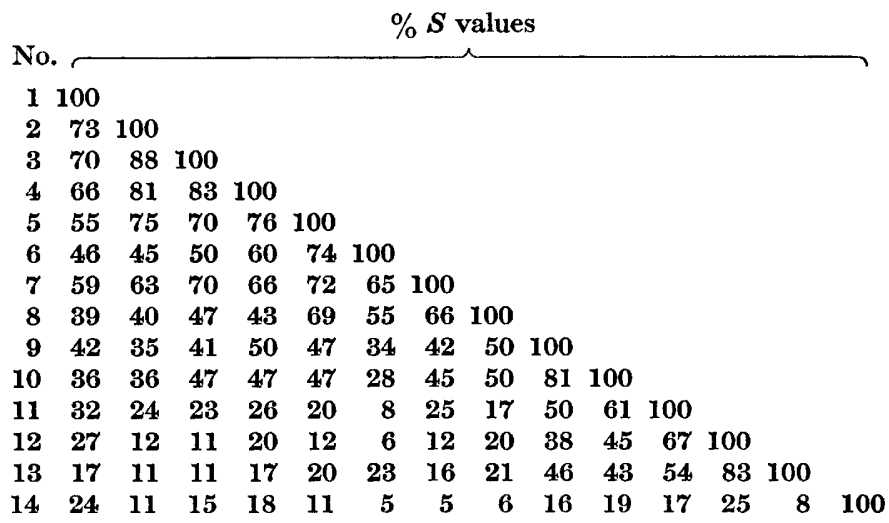

Group of chromobacteria. The $S$ values were taken from Sneath (1957b, Table 7). These values are based on a wide range of tests in which the bias was as far as possible eliminated, and they therefore represent taxonomic relations which are considered to be valid. The model shown (Pl. 2, figs. 3, 4) was made with distance $d$ proportional to $(1 / S)-1$ (see below).

Conversion of $\mathrm{S}$ values into distance in the models. To convert $S$ values into distances several different conventions are possible. The simplest is to make the distance, $d$, between two strains proportional to $1-S$, when $S$ is the $S$-value between the two strains. This has the disadvantage that parts of the model may be separated by distances which represent a smaller similarity than $0 \%$. To avoid this one may make the distance between strains proportional to $(1 / S)-1$, since the distance which corresponds to $0 \%$ similarity is infinity; this convention exaggerates the low similarities. Other conventions, such as making the distance proportional to the logarithm of $(1 / S)$ may be used. We do not consider that any of these conventions has any special taxonomic significance or validity; they are simply conventions which are useful for certain purposes. The convention which probably best represents taxonomic relations is a form of the Generalized Distance (see Rao, 1948) in which generalized distance is equal to $n_{d}$ in the symbols of Sneath $(1957 b)$. This is a convention which uses multidimensional Euclidean space, but since the distance $d$ depends on the number of features examined, it must for useful results be converted into some form of relative distance which is theoretically independent mathematically of the number of features studied. There are other difficulties in using this convention, such as the uncertainty of the distance $d$ 
which is produced by $\mathrm{NC}$ entries in the data; we have therefore not applied it in the present paper.

Form of the models. The most convenient materials are rubber balls held together by lengths of brass rod (brass pinwire, 1-2 mm. diameter). Cork balls may be used but they are apt to break up. Soft copper or iron wire is too pliable for use. The pinwire was cut into lengths determined by the $S$ values between the bacteria and the convention employed for representing $S$. The ends of the pinwire were pushed to the centre of the balls. Sometimes when the balls are very close it is necessary to slice away part of them to get their centres the correct distance apart. Each bacterium (each group of enterobacteria in the first model, or each strain in the second model) was represented by one ball. It was found convenient to choose three or four well-separated bacteria as a locus around which to add the other bacteria. As mentioned in the Discussion, some distortion of the $S$ values is inevitable in making a model; this was kept to a minimum by trial and error during the building of the models.

\section{RESULTS}

Enterobacteria. It is seen from $\mathbf{P l}$. 1, figs 1, 2, that the model may be divided into three main parts: (1) that containing Proteus sensu latu; (2) that containing Shiga's bacillus; (3) that containing the remainder of the groups. It seems very probable, in view of the intermediate forms which occur between Shigella and Escherichia and the fact that they can undergo genetic exchange (Luria \& Burrous, 1957), that the other members of Shigella would be interforms connecting Shiga's bacillus to group (3) if they were included in this model.

The close relation of Salmonella to Arizona is well shown, as are the close relations of Proteus vulgaris to Proteus mirabilis, and of Rettgerella to Providencia. These relations are not unexpected, since this is a model of current ideas of the taxonomy of the family, and not necessarily of the best general taxonomy. In Table 3 are given the Cartesian co-ordinates of this model, from which it can be reconstructed.

In the chromobacteria ( $\mathrm{Pl} .2$, figs. 3, 4) it is seen that there are two large but distinct clusters of balls, representing the mesophilic and the psychrophilic groups of Chromobacterium. Aberrant and atypical strains are represented by outlying balls. The other strains are chromogenic bacteria which are distinct from the genus Chromobacterium itself. At the top are two organisms which seem more closely allied to the Gram-positive bacteria. Strains of Pseudomonas and of Serratia are also shown. Serratia is represented in both models, and this shows roughly how the enterobacteria and chromobacteria are related to one another.

If we had used a number of strains of each group of the enterobacteria we would have obtained clusters of balls which would be centred around the points at which the single balls are present in the first model. These single balls represent typical strains (modoforms) of the groups. It is likely that the clusters would have overlapped a good deal. 
Table 3. Cartesian co-ordinates of the model of enterobacteria

These co-ordinates are the distances in $\mathrm{cm}$. of the balls in the model (Pl. 1, figs. 2, 3) given in three axes at right angles to one another. The origin is the ball 6, representing Klebsiella.

\begin{tabular}{|c|c|c|c|c|}
\hline & & & $\begin{array}{l}m \text { the } \\
\text { axes }\end{array}$ & \\
\hline & & $x$ & $y$ & $z$ \\
\hline & & & tances & \\
\hline Group name & No. & 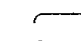 & $\overbrace{-1}$ & -1 \\
\hline Escherichia & 1 & $\mathbf{0}$ & $5 \cdot 9$ & 0 \\
\hline Salmonella & 2 & $3 \cdot 2$ & $4 \cdot 2$ & 0 \\
\hline Arizona & 3 & $3 \cdot 7$ & 5.9 & $1 \cdot 0$ \\
\hline Citrobacter & 4 & $2 \cdot 6$ & $4 \cdot 8$ & $2 \cdot 0$ \\
\hline Cloaca & 5 & $\mathbf{3} \cdot \mathbf{1}$ & $\mathbf{2 \cdot 2}$ & $1 \cdot 4$ \\
\hline Klebsiella & 6 & 0 & 0 & 0 \\
\hline Hafnia & 7 & $2 \cdot 8$ & $0 \cdot 1$ & $2 \cdot 8$ \\
\hline Serratia & 8 & 4.9 & $2 \cdot 6$ & $4 \cdot 6$ \\
\hline Proteus vulgaris & 9 & $1 \cdot 1$ & $5 \cdot 0$ & $6 \cdot 6$ \\
\hline P. mirabilis & 10 & $0 \cdot 6$ & $3 \cdot 3$ & $5 \cdot 9$ \\
\hline Morganella & 11 & $-2 \cdot 6$ & $3 \cdot 3$ & $5 \cdot 6$ \\
\hline Providencia & 12 & $-4 \cdot 1$ & $7 \cdot 9$ & $\mathbf{2 \cdot 6}$ \\
\hline Rettgerella & 13 & $-4 \cdot 3$ & $6 \cdot 4$ & $3 \cdot 8$ \\
\hline Shigella type A1 & 14 & $-0 \cdot 1$ & $12 \cdot 5$ & $2 \cdot 2$ \\
\hline
\end{tabular}

The distance, $s$, between two points is given by the formula $d^{2}=x^{2}+y^{2}+z^{2}$, where $x, y$ and $z$ are the algebraic differences between the co-ordinates of the two points in the $x, y$ and $z$ axes respectively.

\section{DISCUSSION}

The older schemes of bacterial taxonomy may be more of a handicap than a help in the future. A new general taxonomy of bacteria has not yet been developed (though attempts are now being made in this direction; see Sneath \& Cowan, 1958), and models such as those shown here may be of help in obtaining a general orientation when building such a taxonomy. One of the greatest difficulties is the lack of comparative bacteriology, since much of our knowledge comes from specialities such as dairy microbiology and soil microbiology, and it seems probable that many bacteria known by different names to workers in different specialities are in fact identical. A classic case is in Pseudomonas, where it is very likely that some of the species which are pathogenic for plants are identical with Pseudomonas aeruginosa from animals (see Elrod \& Braun, 1942). It is also not unusual to be unable to identify a well-known bacterium when it has been isolated from an unusual habitat.

The biological relationships of organisms are of course not the same as the mathematical relations in these models, since the latter are only summaries, incomplete and distorted, of the former. This distortion is inevitable because, as has been mentioned in the description of the method, the relations between organisms are multidimensional, with one dimension for every feature which can vary independently of the rest. This compression, with subsequent distortion, into two dimensions (as in taxonomic trees) or into three (as in the 
models) is, however, essential for handling and publishing taxonomic data. It would ideally be carried out by the mathematical procedure of Principle Component Analysis.

The value of the models is that they allow better expression of relations than is afforded by two dimensions, they are useful in obtaining a preliminary idea of the taxonomy of a group under study and they are very suitable for teaching. Intermediate strains (interforms) in such models are placed between the clusters which represent the more typical members of the groups under study. One other useful property is that, by colouring or labelling the balls, the reactions of the strains in a few tests which are of special concern to the worker in diagnostic or epidemiological work can strikingly be shown.

The degree to which the $S$ values are distorted on reducing them from a multidimensional quantity to one in three dimensions does not seem, in the present examples at any event, to be very great. This was checked in the first model by measuring all the distances between bacteria and comparing them with the $S$ value table (the actual distances may be derived from Table 3). The great majority of such distances were close to those implied by the $S$ values, though in a few the differences were over $2.5 \mathrm{~cm}$. (corresponding to $25 \% S$ ). In the best possible model these discrepancies would be as small as possible.

\section{REFERENCES}

Cowan, S. T. (1956). Taxonomic rank of Enterobacteriaceae 'groups'. J. gen. Microbiol. 15, 345.

Davis, B. R., Ewing, W. H. \& Reavis, R. W. (1957). The biochemical reactions given by members of the Serratia group. Internat. Bull. bact. Nomen. Taxon. 7, 151.

Elrod, R. P. \& Braun, A. C. (1942). Pseudomonas aeruginosa: its role as a plant pathogen. J. Bact. 44, 633.

KaUfFManN, F. (1956). A simplified biochemical table of Enterobacteriaceae. Acta path. microbiol. scand. 39, 103.

Luria, S. E. \& Burrous, J. W. (1957). Hybridization between Escherichia coli and Shigella. J. Bact. 74, 461.

Møluer, V. (1955). Simplified tests for some amino acid decarboxylases and for the arginine dihydrolase system. Acta path. microbiol. scand. 36, 158.

RAo, C. R. (1948). The utilization of multiple measurements in problems of biological classification. J. R. statist. Soc. B, 10, 159.

SNeath, P. H. A. $(\mathbf{1 9 5 7} a)$. Some thoughts on bacterial classification. J. gen. Microbiol. 17, 184.

Sneath, P. H. A. $(1957 b)$. The application of computers to taxonomy. J. gen. Microbiol. 17, 201.

Sneath, P. H. A. \& Cowan, S. T. (1958). An electrotaxonomic survey of bacteria. J. gen. Microbiol. 19, 551. 


\section{EXPLANATION OF PLATES}

Plate 1

Fig. 1. Taxonomic model of the Enterobacteriaceae. The numbers and names where shown are those used for the groups in Table 2.

Fig. 2. Taxonomic model of the Enterobacteriaceae: another view.

\section{Plate 2}

Fig. 3. Taxonomic model of chromobacteria. The groups of strains were as follows : $(a)=$ mesophilic strains of Chromobacterium (C. violaceum); $(b)=$ psychrophilic strains of Chromobacterium (C. lividum); (c)=Serratia; $(d)=$ Pseudomonas aeruginosa; $(e)=$ 'Chromobacterium viscosum'; $(f)=$ 'Chromobacterium iodinum'; $(g)=$ 'Chromobacterium ianthinum'.

Fig. 4. Taxonomic model of chromobacteria: another view.

(Received 16 October 1958) 
Journal of General Microbiology, Vol. 20, No. 2

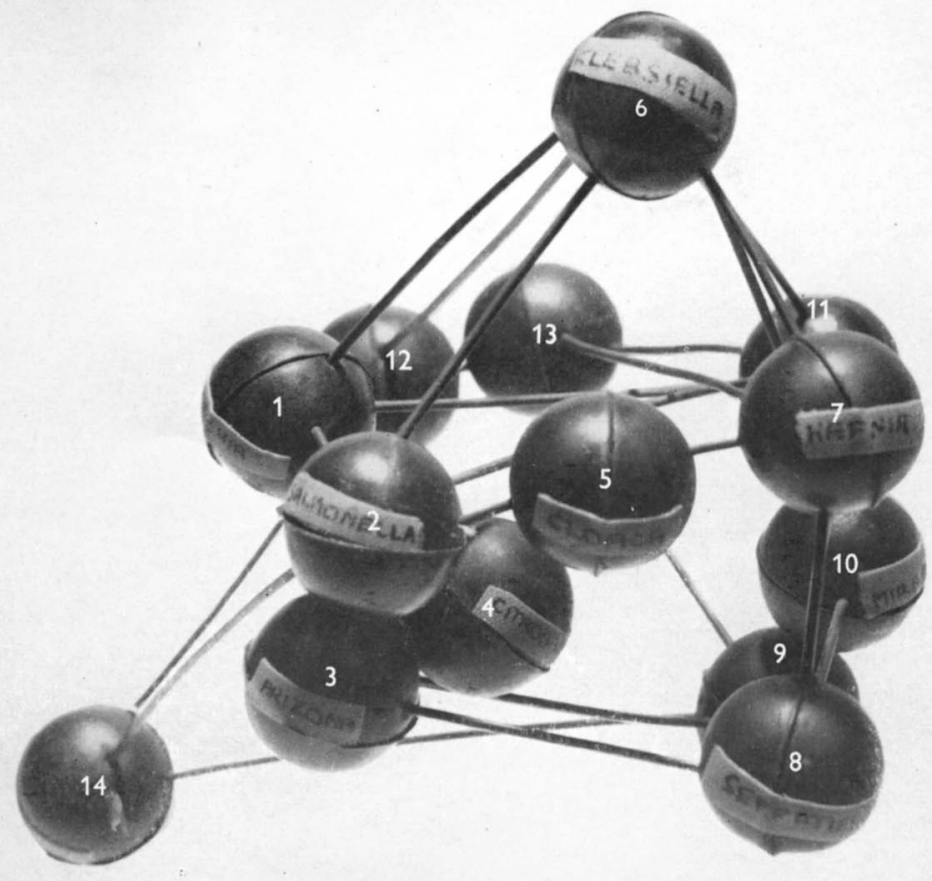

Fig. 1

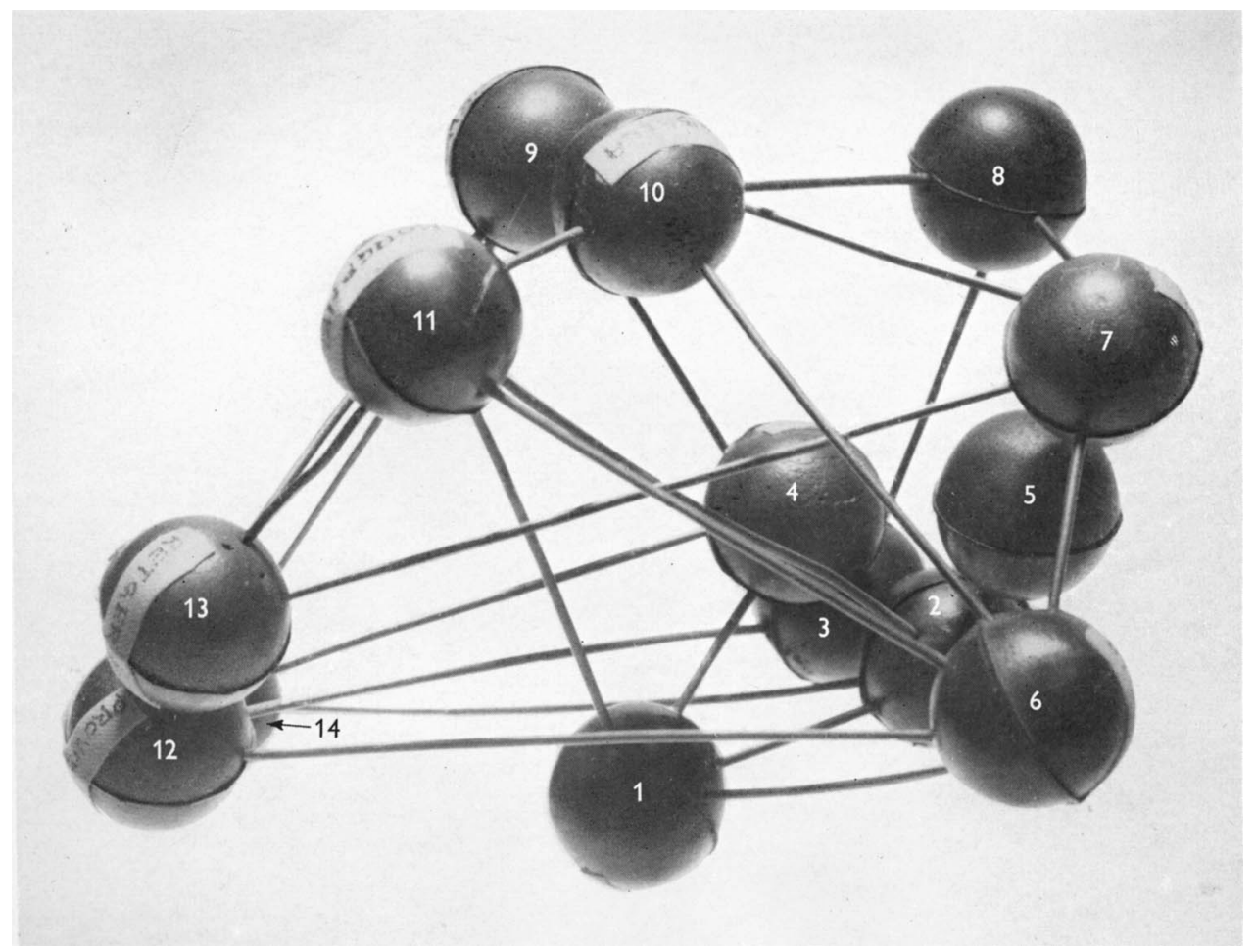

Fig. 2

O. Lysenko \& P. H. A. Sneath-Models in taxonomy. Plate 1 
Journal of General Microbiology, Vol. 20, No. 2

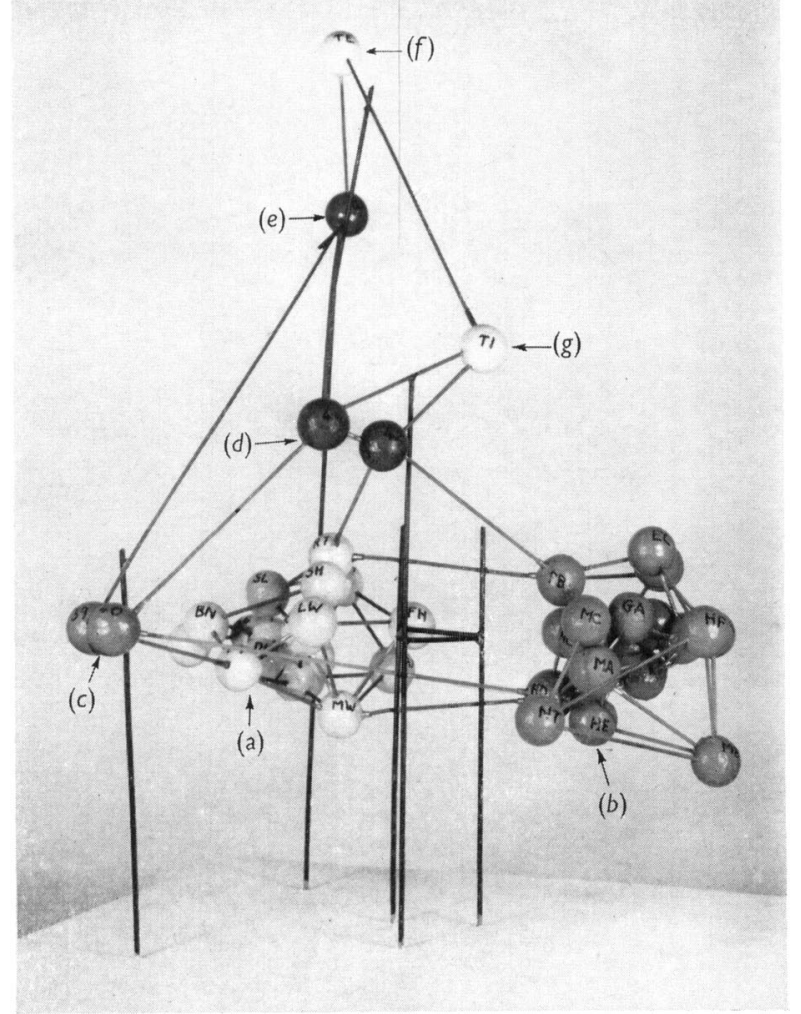

Fig. 3

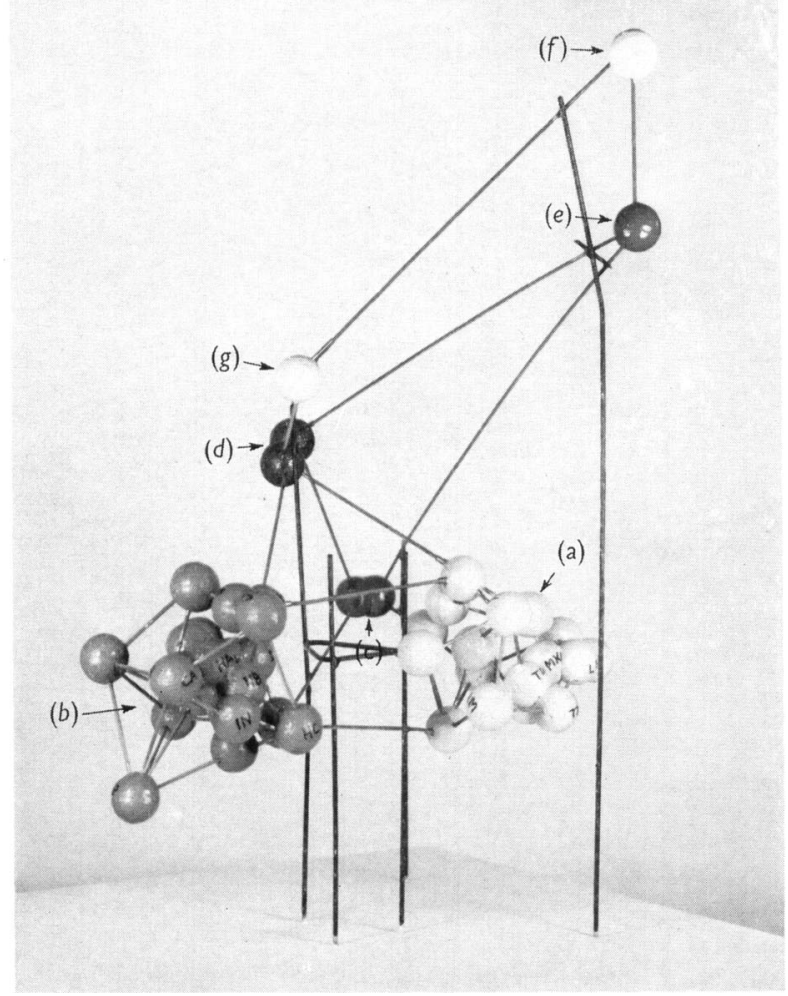

Fig. 4

O. Lysenko \& P. H. A. Sneati-Mobels in taxonomy. Plate 2 